CRYSTALLOGRAPHIC COMMUNICATIONS
Received 22 January 2020

Accepted 16 March 2020

Edited by C. Massera, Università di Parma, Italy

Keywords: crystal structure; anti-TB activity drug; intermolecular interactions; Hirshfeld surface analysis; fingerprint plot.

CCDC reference: 1865697

Supporting information: this article has supporting information at journals.iucr.org/e

\section{Structural investigation of methyl 3-(4-fluoro- benzoyl)-7-methyl-2-phenylindolizine-1-carboxyl- ate, an inhibitory drug towards Mycobacterium tuberculosis}

\author{
Avantika Hasija, ${ }^{a}$ Subhrajyoti Bhandary, ${ }^{a}$ Katharigatta N. Venugopala,, ${ }^{b, c}$ Sandeep \\ Chandrashekharappa $^{d}$ and Deepak Chopra ${ }^{\mathrm{a} *}$
}

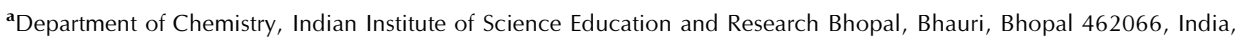
${ }^{\mathbf{b}}$ Department of Pharmaceutical Sciences, College of Clinical Pharmacy, King Faisal University, Al-Ahsa 31982, Kingdom of Saudi Arabia, ' Department of Biotechnology and Food Technology, Durban University of Technology, Durban 4001, South Africa, and $\mathbf{d}^{\mathbf{d} n s t i t u t e}$ for Stem Cell Biology and Regenerative Medicine, NCBS, TIFR, GKVK, Bellary Road,

Bangalore 560 065, India. *Correspondence e-mail: dchopra@iiserb.ac.in

The title compound, $\mathrm{C}_{24} \mathrm{H}_{18} \mathrm{FNO}_{3}$, crystallizes in the monoclinic centrosymmetric space group $P 2_{1} / n$ and its molecular conformation is stabilized via $\mathrm{C}-\mathrm{H} \cdots \mathrm{O}$ intramolecular interactions. The supramolecular network mainly comprises $\mathrm{C}-$ $\mathrm{H} \cdots \mathrm{O}, \mathrm{C}-\mathrm{H} \cdots \mathrm{F}$ and $\mathrm{C}-\mathrm{H} \cdots \pi$ interactions, which contribute towards the formation of the crystal structure. The different intermolecular interactions have been further analysed via Hirshfeld surface analysis and fingerprint plots.

\section{Chemical context}

Indolizine represents an interesting heterocyclic scaffold in which the nitrogen atom belongs to both of the fused six- and five-membered rings. It is a well-known pharmacophore endowed with various promising pharmacological properties. For instance, indolizines have been found to exhibit analgesic (Vaught et al., 1990), anticancer (Butler, 2008; Sandeep et al., 2016a,b), antidiabetic (Mederski et al., 2012), antihistaminic (Cingolani et al., 1990), anti-microbial (Hazra et al., 2011) and antiviral (Mishra \& Tiwari, 2011) activity. It has also been found to act as cyclo-oxygenase (COX-2) inhibitor (Chandrashekharappa et al., 2018b) and to have larvicidal activity against Anopheles arabiensis (Chandrashekharappa et al., 2018a).

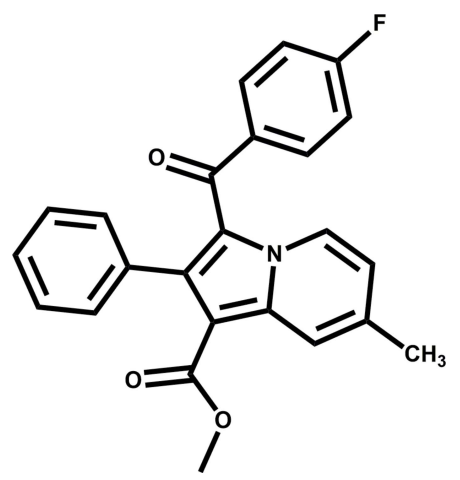

The title compound, comprising a substituted indolizine unit, displays a modest activity against susceptible H37Rv strains of Mycobacterium tuberculosis (Venugopala et al., 2019). Besides the tremendous scope of the pharmacological studies on indolizine-based compounds, the substitution of 


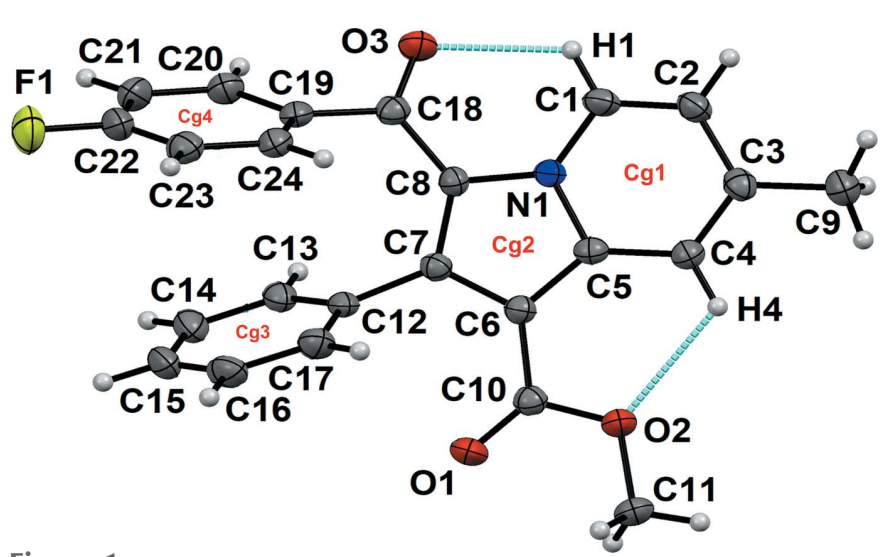

Figure 1

Ellipsoid plot of the title compound drawn with $50 \%$ probability ellipsoids. Dotted lines indicate intramolecular $\mathrm{C}-\mathrm{H} \cdots \mathrm{O}$ interactions. $C g 1, C g 3$ and $C g 4$ represent the centroids of the six-membered rings N1/ $\mathrm{C} 1-\mathrm{C} 5, \mathrm{C} 12-\mathrm{C} 17$ and $\mathrm{C} 18 / \mathrm{O} 3 / \mathrm{C} 19-\mathrm{C} 24 / \mathrm{F} 1$, respectively, while $\mathrm{Cg} 2$ represents the five-membered ring N1/C5-C8.

fluorine on the benzoyl ring, the presence of flexible moieties and of competitive hydrogen-bond acceptors (namely, oxygen $\mathrm{O} 2$ in the ester group at $\mathrm{C} 6$ and $\mathrm{O} 3$ in the carbonyl group at C8) make the structural study of the title compound of extreme relevance. In addition, it is of importance to observe the cooperative interplay of weak interactions that contribute towards the consolidation of the crystal lattice. In the present paper, we report the molecular and crystal structure of the title compound, highlighting its molecular conformation and analysing the different intermolecular interactions via Hirshfeld surface analysis and fingerprint plots.

\section{Structural commentary}

The title compound crystallizes in the centrosymmetric monoclinic $P 2_{1} / n$ space group. The molecular structure

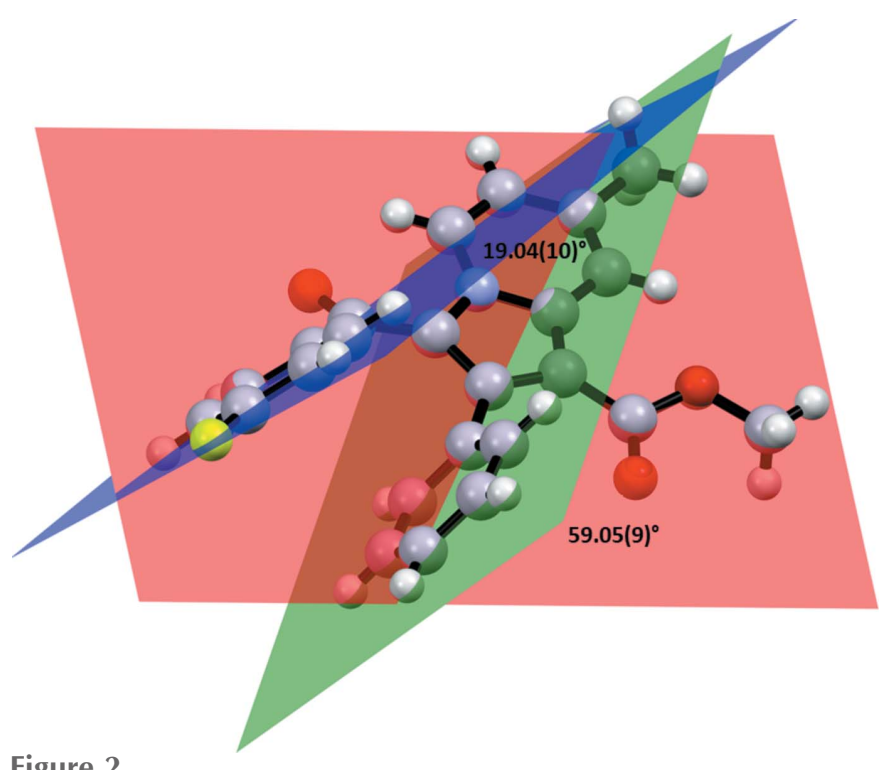

Figure 2

Dihedral angles between the mean plane passing through the $\mathrm{C} 12-\mathrm{C} 17$ ring (green) and the $\mathrm{C} 18 / \mathrm{O} 3 / \mathrm{C} 19-\mathrm{C} 24 / \mathrm{F} 1$ ring (blue) and through the indolizine skeleton (red).
Table 1

Hydrogen-bond geometry $\left(\AA,^{\circ}\right)$.

\begin{tabular}{lllll}
\hline$D-\mathrm{H} \cdots A$ & $D-\mathrm{H}$ & $\mathrm{H} \cdots A$ & $D \cdots A$ & $D-\mathrm{H} \cdots A$ \\
\hline $\mathrm{C} 1-\mathrm{H} 1 \cdots \mathrm{O} 3$ & 0.95 & 2.26 & $2.853(3)$ & 120 \\
$\mathrm{C} 4-\mathrm{H} 4 \cdots \mathrm{O} 2$ & 0.95 & 2.38 & $2.927(2)$ & 116 \\
$\mathrm{C} 21-\mathrm{H} 21 \cdots \mathrm{O}^{\mathrm{i}}$ & 0.95 & 2.54 & $3.399(3)$ & 149 \\
$\mathrm{C} 2-\mathrm{H} 2 \cdots 1^{\mathrm{ii}}$ & 0.95 & 2.63 & $3.531(4)$ & 157 \\
$\mathrm{C} 15-\mathrm{H} 15 \cdots \mathrm{O}^{\mathrm{iii}}$ & 0.95 & 2.76 & $3.519(4)$ & 137 \\
$\mathrm{C} 1-\mathrm{H} 1 \cdots \mathrm{C} 15^{\text {ii }}$ & 0.95 & 2.74 & $3.6064(3)$ & 152 \\
$\mathrm{C} 11-\mathrm{H} 11 A \cdots \mathrm{C}^{\text {iv }}$ & 0.98 & 2.74 & $3.4906(1)$ & 133 \\
$\mathrm{C} 11-\mathrm{H} 11 B \cdots 1^{v}$ & 0.98 & 2.67 & $3.0585(3)$ & 104 \\
$\mathrm{C} 23-\mathrm{H} 23 \cdots \mathrm{O}^{\mathrm{vi}}$ & 0.95 & 2.67 & $3.4875(3)$ & 143 \\
\hline
\end{tabular}

Symmetry codes: (i) $-x+\frac{3}{2}, y+\frac{1}{2},-z+\frac{1}{2}$; (ii) $x, y-1, z$; (iii) $x, y+1, z$; (iv) $-x,-y+1,-z$; (v) $x-\frac{1}{2},-y+\frac{3}{2}, z-\frac{1}{2}$; (vi) $-x+\frac{1}{2}, y+\frac{1}{2},-z+\frac{1}{2}$.

comprises one methylindolizine heterocyclic moiety (N1/C1C9) consisting of fused six- and five-membered rings (N1/C1$\mathrm{C} 5$, centroid $C g 1$ and N1/C5-C8, centroid Cg2). The heterocycle is substituted at the carbon atoms $\mathrm{C} 6, \mathrm{C} 7$ and $\mathrm{C} 8$ with a methoxy carbonyl group, a phenyl ring (C12-C17, centroid Cg3), and a fluorobenzoyl ring [C18/O3/C19-C24/F1, centroid Cg4], respectively (Fig. 1). The molecular structure possesses three conformational degrees of freedom due to the free rotation with respect to the $\mathrm{C} 6-\mathrm{C} 10, \mathrm{C} 7-\mathrm{C} 12$, and $\mathrm{C} 8-\mathrm{C} 18$ single bonds. The molecular conformation is stabilized by the presence of intramolecular $\mathrm{C} 1-\mathrm{H} 1 \cdots \mathrm{O} 3[\mathrm{C} 1 \cdots \mathrm{O} 3=$ $2.853(3) \AA]$ and $\mathrm{C} 4-\mathrm{H} 4 \cdots \mathrm{O} 2 \quad[\mathrm{C} 4 \cdots \mathrm{O} 2=2.927(2) \AA]$ interactions (Table 1 ) and by $\pi-\pi$ stacking $[\mathrm{Cg} 3 \cdots \mathrm{Cg} 4=$ 3.5084 (13) A]. The dihedral angle between the mean plane through ring $C g 3$ (coloured in green in Fig. 2) and the mean plane of the indolizine skeleton (coloured in red) is $59.05(9)^{\circ}$, while the dihedral angle between the mean plane through the phenyl ring and that through the fluorobenzoyl ring (coloured in blue) is as small as $19.04(10),{ }^{\circ}$ showing the nearly parallel position of the rings. The torsion angles $\mathrm{N} 1-\mathrm{C} 8-\mathrm{C} 18-\mathrm{C} 19$ and $\mathrm{C} 8-\mathrm{C} 18-\mathrm{C} 19-\mathrm{C} 24$ are $-161.74(19)$ and $46.2(3)^{\circ}$, respectively.

\section{Supramolecular features}

The list of all intra- and intermolecular interactions along with their geometrical parameters have been reported in Table 1. The interactions included for investigation are based on the distance criteria of vdW $+0.4 \AA$ (Dance, 2003). In the crystal, the molecules are primarily assembled through concomitant $\mathrm{C} 2 / 15-\mathrm{H} 2 / 15 \cdots \mathrm{O} 1^{\mathrm{ii}} / \mathrm{O} 3^{\mathrm{iii}}$ interactions $\left[\mathrm{C} 2 \cdots \mathrm{O} 1^{\mathrm{ii}}=\right.$ 3.531 (4) $\AA$, $157^{\circ}$; $\mathrm{C} 15 \cdots \mathrm{O} 3^{\mathrm{iii}}=3.519$ (4) $\AA$, $137^{\circ}$; symmetry codes: (ii) $x, y-1, z$; (iii) $x, y+1, z]$ and $\mathrm{C} 1-\mathrm{H} 1 \cdots \pi(\mathrm{C} 15)^{\mathrm{ii}}$ $\left[\mathrm{C} 1 \cdots \mathrm{C} 15=3.6064\right.$ (3) $\AA$, $152^{\circ}$ ], forming ribbons along the [010] direction, as shown by the green shading in Fig. 3. Two adjacent ribbons are connected to each other via $\mathrm{C} 11-$ $\mathrm{H} 11 B \cdots \mathrm{F} 1^{\mathrm{v}}\left[\mathrm{C} 11 \cdots \mathrm{F} 1=3.0585(3) \AA\right.$ А , 104 ${ }^{\circ}$; symmetry code: (v) $\left.x-\frac{1}{2},-y+\frac{3}{2}, z-\frac{1}{2}\right]$ (Fig. 3) and $\mathrm{C} 21-\mathrm{H} 21 \cdots \mathrm{O} 3^{\mathrm{i}}$

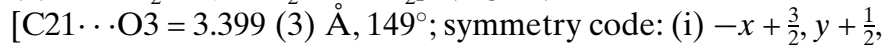
$-z+\frac{1}{2}$ ] (Fig. 4) interactions in a zigzag fashion along [001], resulting in the formation of a molecular sheet parallel to the ac plane. Analogous $\mathrm{C}-\mathrm{H} \cdot \mathrm{F}$. interactions have been investigated, showing that where the angularity is in the range 90 to 


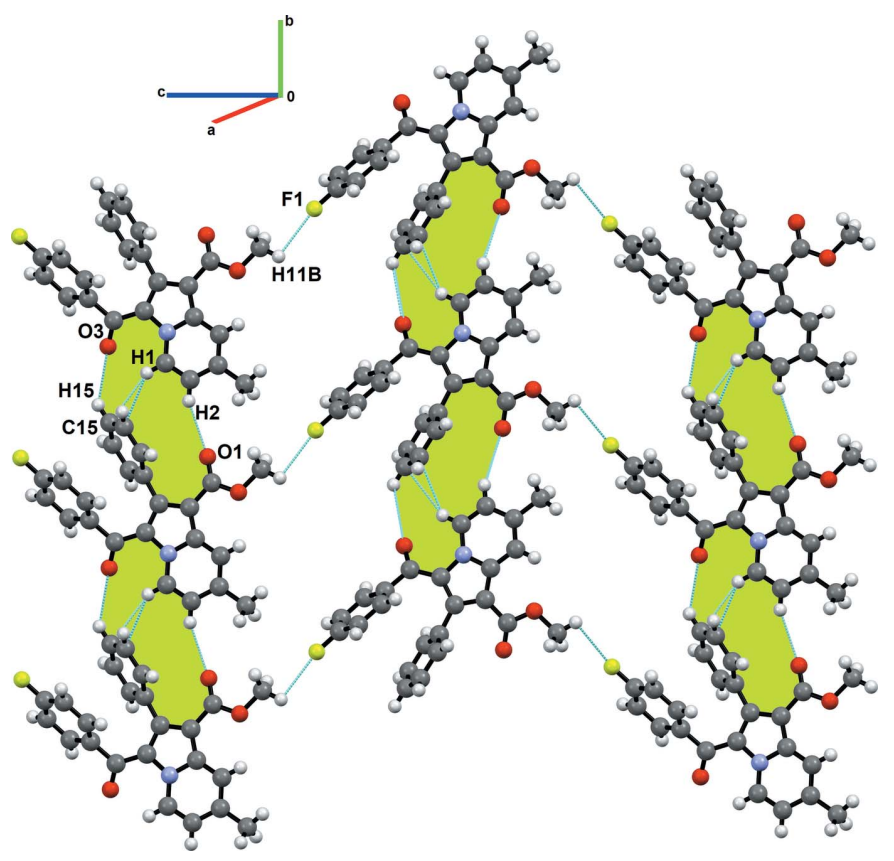

Figure 3

Crystal packing of title compound showing the formation of molecular sheets parallel to the $b c$ plane via $\mathrm{C}-\mathrm{H} \cdots \mathrm{O}, \mathrm{C}-\mathrm{H} \cdots \pi$ and $\mathrm{C}-\mathrm{H} \cdots \mathrm{F}$ interactions.

$140^{\circ}$, the $\sigma$-hole on fluorine is directed towards the electron density of the $\mathrm{C}-\mathrm{H}$ bond (Hathwar et al., 2020), underlining the importance of interactions with low angularity. The molecular sheets are closely stacked along the $a$-axis direction via weak interactions such as $\mathrm{C} 9-\mathrm{H} 9 \mathrm{C} \cdots \pi(\mathrm{C} 1)\left[\mathrm{C} 9 \cdots \mathrm{C} 1^{\mathrm{vii}}=\right.$ 3.7431 (5) А; symmetry code: (vii) $-x+1,-y,-z], \mathrm{C} 11-$ $\mathrm{H} 11 A \cdots \pi(\mathrm{C} 5)\left[\mathrm{C} 11 \cdots \mathrm{C} 5^{\mathrm{iv}}=3.4906(4) \AA\right.$; symmetry code: (iv) $-x,-y+1,-z], \mathrm{C} 11-\mathrm{H} 11 C \cdots \pi(\mathrm{C} 8)\left[\mathrm{C} 11 \cdots \mathrm{C} 8^{\text {viii }}=\right.$ 3.6590 (5) Å; symmetry code: (viii) $-x+1,-y+1,-z]$ (Fig. 4), giving rise to a layered supramolecular structure. From this analysis, it can be stated that the formation of the crystal structure is mainly governed by several $\mathrm{C}-\mathrm{H} \cdots \mathrm{O}$ and $\mathrm{C}-$ $\mathrm{H} \cdots \pi$ interactions, while the $\mathrm{C}-\mathrm{H} \cdots \mathrm{F}$ interactions play a secondary but supporting role in its overall consolidation.

\section{Database survey}

A search for the 2-phenylindolizine skeleton in the CSD (version 5.40, update of August 2019; Groom et al., 2016) was carried out. Out of the 39 hits for unsubstituted phenyl rings attached to indolizine, the majority of entries gave reports of varied synthetic procedures and methodologies to obtain these compounds, underlining their importance. The nearinfrared emissive properties of KIVLIN, KIVLOT, KIVLUZ (Gayton et al., 2019) and KENFAN (McNamara et al., 2017) have also been reported.

Structural details of compounds such as CAJTAI (Aslanov et al., 1983), EMUTOV (Liu, et al., 2003), FEDQAH (Liu, et al., 2005), GIYLOP (Sonnenschein \& Schneider, 1997), ODEFIN (Qian et al., 2006), PNOIZA, PNOIZB, PNOIZE, PNOIZF (Tafeenkov \& Aslanov, 1980), ROLKIM (Tafeenkov \& Au, 1996) and TIGXOX (Liu, et al., 2007) have also been deposited. Almost all of these molecules are substituted at the C8 position with electron-withdrawing substituents such as $-\mathrm{COMe},-\mathrm{CH}_{2} \mathrm{CN},-\mathrm{CN},-\mathrm{N}=\mathrm{O},-\mathrm{CH}=\mathrm{C}(\mathrm{Ph})(\mathrm{CN})$, etc.

In particular, the papers reporting TIGXOX (Liu et al., 2007), FEDQAH (Liu et al., 2005) and ODEFIN (Qian et al., 2006) discuss the structural features of molecules comprising the 2-phenyl indolizine skeleton, showing high fluorescent efficiency. In these reports, the respective dihedral angles between the mean plane of the indolizine skeleton and the plane of the phenyl ring are $c a 53,39$ and 49 and $45^{\circ}$, comparable to that reported in the title compound.

\section{Hirshfeld surface analysis and fingerprint plots}

The significance of the cumulative effect of the interactions involved in the crystal structure can be visualized qualitatively through Hirshfeld surface analysis (Spackman et al., 2009). The Hirshfeld surfaces and the two-dimensional fingerprint plots were calculated using CrystalExplorer (Version 17.5; Wolff et al., 2012) and are shown in Figs. 5 and 6, respectively. The red spots on the HS surface illustrate the presence of

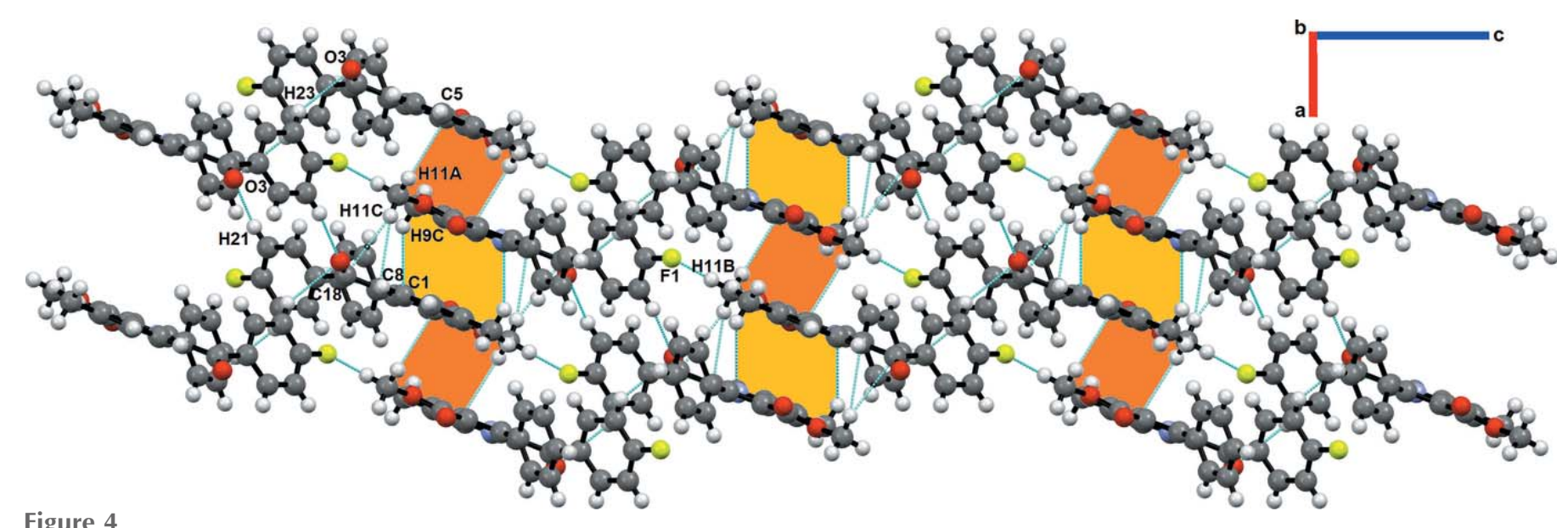

Figure 4

Stacking of molecular sheets along the $a$-axis direction, primarily via $\mathrm{C}-\mathrm{H} \cdots \pi$ and $\mathrm{C}-\mathrm{H} \cdots \mathrm{F}$ interactions, resulting in a layered supramolecular architecture. 

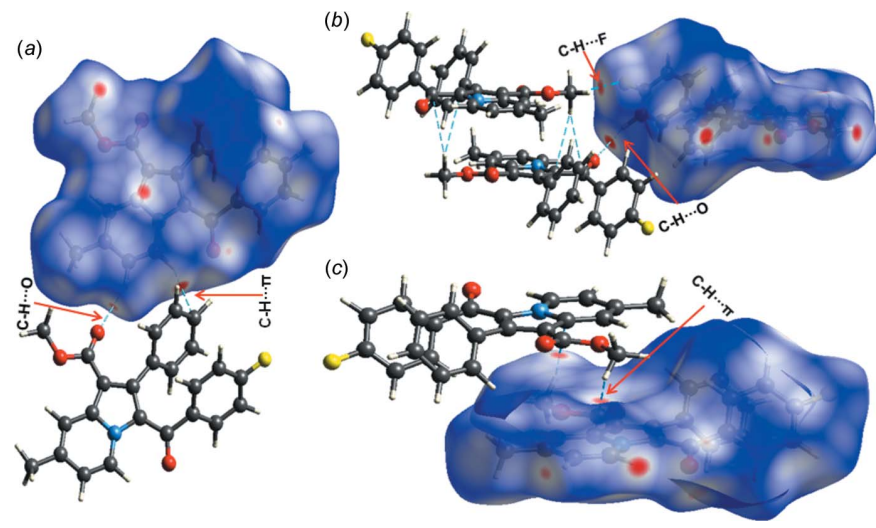

Figure 5

The Hirshfeld surface of title compound mapped over $d_{\text {norm. }}$. Dashed lines indicate hydrogen bonds.

supramolecular interactions such as $\mathrm{C}-\mathrm{H} \cdots \mathrm{O}, \mathrm{C}-\mathrm{H} \cdots \pi$ and $\mathrm{C}-\mathrm{H} \cdots \mathrm{F}$ whereas the blue regions indicate the lack of contact distances shorter than the sum of the van der Waals radii. The fingerprint plots represent the individual contributions of the different interactions. Fig. 6 shows that the major contribution comes from $\mathrm{H} \cdots \mathrm{H}(47.1 \%), \mathrm{O} \cdots \mathrm{H} / \mathrm{H} \cdots \mathrm{O}$ $(13.1 \%), \mathrm{C} \cdots \mathrm{H} / \mathrm{H} \cdots \mathrm{C}(21.4 \%), \mathrm{H} \cdots \mathrm{F} / \mathrm{F} \cdots \mathrm{H}(9.0 \%), \mathrm{C} \cdots \mathrm{C}$ $(1.9 \%)$ and $\mathrm{N} \cdots \mathrm{H} / \mathrm{H} \cdots \mathrm{N}(1.7 \%)$ contacts. The relatively high percentage of $\mathrm{C} \cdots \mathrm{H} / \mathrm{H} \cdots \mathrm{C}$ contacts indicates how the contribution of all of the $\mathrm{C}-\mathrm{H} \cdots \pi$ interactions plays an important role in consolidating the crystal packing.

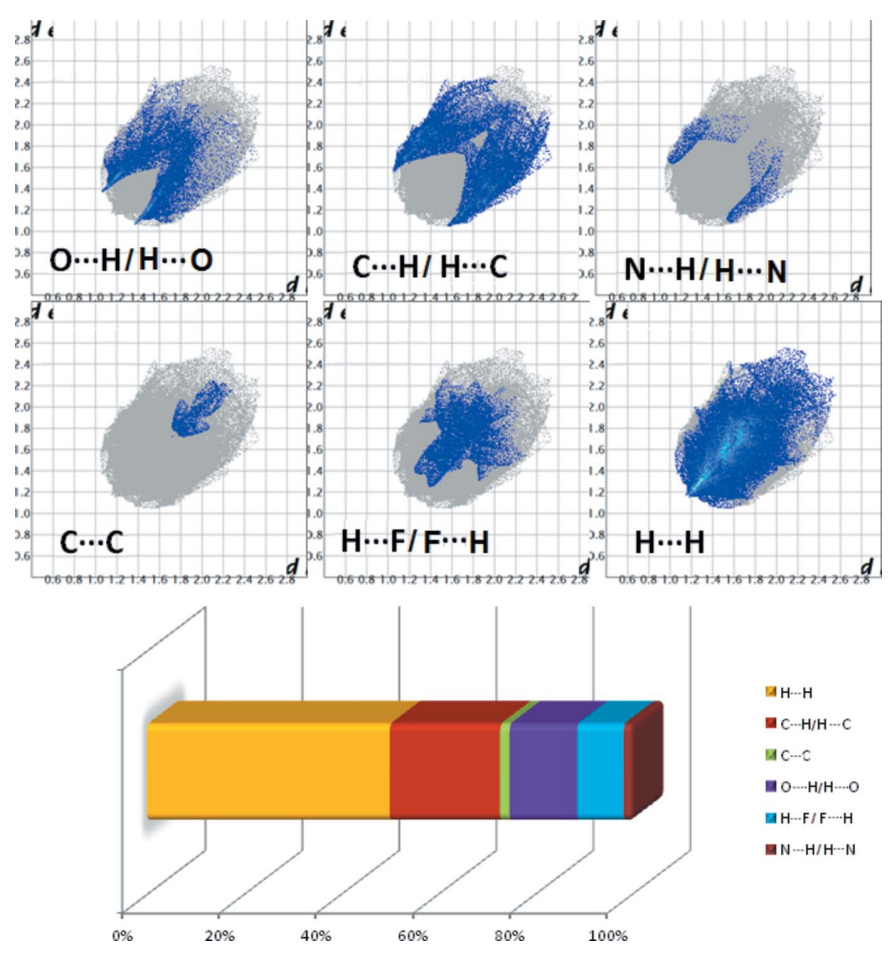

Figure 6

The fingerprint plots of the title compound showing the different contributions deriving from the $\mathrm{O} \cdots \mathrm{H} / \mathrm{H} \cdots \mathrm{O}, \mathrm{N} \cdots \mathrm{H} / \mathrm{H} \cdots \mathrm{N}, \mathrm{C} \cdots \mathrm{H} /$ $\mathrm{H} \cdots \mathrm{C}, \mathrm{H} \cdots \mathrm{F} / \mathrm{F} \cdots \mathrm{H}, \mathrm{C} \cdots \mathrm{C}$ and $\mathrm{H} \cdots \mathrm{H}$ contacts.

Table 2

Experimental details.

Crystal data

Chemical formula

$M_{\text {r }}$

Crystal system, space group

Temperature (K)

$a, b, c(\AA)$

$\beta\left({ }^{\circ}\right)$

$V\left(\AA^{3}\right)$

Z

Radiation type

$\mu\left(\mathrm{mm}^{-1}\right)$

Crystal size (mm)

Data collection

Diffractometer

Absorption correction

$T_{\min }, T_{\max }$

No. of measured, independent and observed $[I>2 \sigma(I)]$ reflections

$R_{\text {int }}$

$(\sin \theta / \lambda)_{\max }\left(\AA^{-1}\right)$

Refinement

$R\left[F^{2}>2 \sigma\left(F^{2}\right)\right], w R\left(F^{2}\right), S$

No. of reflections

No. of parameters

$\mathrm{H}$-atom treatment

$\Delta \rho_{\max }, \Delta \rho_{\min }\left(\mathrm{e} \AA^{-3}\right)$

Computer programs: APEX2 (Bruker, 2012), SAINT (Bruker, 2008), SHELXS97 (Sheldrick, 2008), X-SEED (Barbour, 2001), Mercury (Macrae et al., 2020), SHELXL2014 (Sheldrick, 2015) and PLATON (Spek, 2020).

\section{Synthesis and crystallization}

All chemicals were obtained from Sigma-Aldrich and used without further purification. A mixture of methyl 3-phenylpropiolate (1) (160 mg, $1 \mathrm{mmol})$, 4-methylpyridine (2) $(93 \mathrm{mg}$, $1 \mathrm{mmol}), 2$-bromo-1-(4-fluorophenyl)ethan-1-one (3) $(217 \mathrm{mg}$, $1 \mathrm{mmol})$, and triethylamine $(0.101 \mathrm{mg}, 1 \mathrm{mmol})$ in $4.5 \mathrm{~mL}$ of acetonitrile were added to a $10 \mathrm{~mL}$ microwave tube under a nitrogen atmosphere (Fig. 7). A microwave initiator was used to irradiate the reaction mixture at $373 \mathrm{~K}$ for about $5 \mathrm{~min}$. The reaction was monitored via TLC. The solvent was then removed under reduced pressure, the crude residue was diluted with water and the aqueous layer was extracted twice with ethyl acetate, and the combined organic solvent was washed with a brine solution. The organic layer was removed under reduced pressure and the remaining residue was subjected to column chromatography using 60-120 mesh silica gel with an ethyl acetate and hexane solvent system to afford
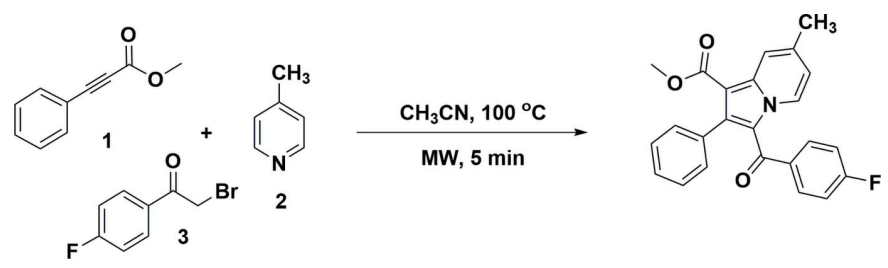

Figure 7

The reaction scheme for the synthesis of the title compound. 
$0.3414 \mathrm{~g} \mathrm{(} 88 \%$ yield) of the title compound (Venugopala et al., 2019). Suitable single crystals of the compound were grown by the slow evaporation of acetone at ambient conditions.

\section{Refinement}

Crystal data, data collection and structure refinement details are summarized in Table 2. The hydrogen atoms were placed in idealized positions and refined using a riding model with $U_{\text {iso }}(\mathrm{H})=1.2 U_{\text {eq }}(\mathrm{C})$ or $1.5 U_{\text {eq }}(\mathrm{C}$-methyl $)$.

\section{Acknowledgements}

The authors are grateful to the Deanship of Scientific Research, King Faisal University, Kingdom of Saudi Arabia for financial support and encouragement. AH thanks IISER Bhopal for a research fellowship. The authors are thankful to the CIF of IISER Bhopal for research facilities and infrastructure.

\section{Funding information}

Funding for this research was provided by: Indian Institute of Science Education and Research Bhopal; the Deanship of Scientific Research, King Faisal University, Kingdom of Saudi Arabia (through Research Group grant No. 17122011).

\section{References}

Aslanov, L. A., Tafeenko, V. A., Paseshnichenko, K. A., Bundel', Y. G., Gromov, S. P. \& Gerasimov, B. G. (1983). Zh. Strukt. Khim. (Russ. J. Struct. Chem.), 24, 427-434.

Barbour, L. J. (2001). J. Supramol. Chem. 1, 189-191.

Bruker (2008). SAINT and SADABS. Bruker AXS Inc., Madison, Wisconsin, USA.

Bruker (2012). APEX2. Bruker AXS Inc., Madison, Wisconsin, USA. Butler, M. S. (2008). Nat. Prod. Rep. 25, 475-516.

Chandrashekharappa, S., Venugopala, K. N., Nayak, S. K., Gleiser, R. M., García, D. A., Kumalo, H. M., Kulkarni, R. S., Mahomoodally, F. M., Venugopala, R., Mohan, M. K. \& Odhav, B. (2018a). J. Mol. Struct. 1156, 377-384.

Chandrashekharappa, S., Venugopala, K. N., Tratrat, C., Mahomoodally, F. M., Aldhubiab, B. E., Haroun, M., Venugopala, R., Mohan, M. K., Kulkarni, R. S., Attimarad, M. V., Harsha, S. \& Odhav, B. (2018b). New J. Chem. 42, 4893-4901.

Cingolani, G. M., Claudi, F., Massi, M. \& Venturi, F. (1990). Eur. J. Med. Chem. 25, 709-712.

Dance, I. (2003). New J. Chem. 27, 22-27.
Gayton, J., Autry, S. A., Meador, W., Parkin, S. R., Hill, G. A. Jr, Hammer, N. I. \& Delcamp, J. H. (2019). J. Org. Chem. 84, 687-697.

Groom, C. R., Bruno, I. J., Lightfoot, M. P. \& Ward, S. C. (2016). Acta Cryst. B72, 171-179.

Hathwar, V. R., Bhowal, R. \& Chopra, D. (2020). J. Mol. Struct. 1208, 1278642 https://doi. org/10.1016/j. molstruc. 2020.127864

Hazra, A., Mondal, S., Maity, A., Naskar, S., Saha, P., Paira, R., Sahu, K. B., Paira, P., Ghosh, S., Sinha, C., Samanta, A., Banerjee, S. \& Mondal, N. B. (2011). Eur. J. Med. Chem. 46, 2132-2140.

Liu, W., Ou, S., He, X., Hu, H., Wu, Q. \& Huang, Z. (2003). J. Chem. Crystallogr. 33, 795-798.

Liu, W.-W., Li, Y.-Z., Sun, R.-K., Hu, H.-W., Wu, Q.-J. \& Huang, Z.-X. (2005). Acta Cryst. E61, o445-o447.

Liu, W.-W., Wang, L., Tang, L.-J., Cao, W. \& Hu, H.-W. (2007). Acta Cryst. E63, o3518.

Macrae, C. F., Sovago, I., Cottrell, S. J., Galek, P. T. A., McCabe, P., Pidcock, E., Platings, M., Shields, G. P., Stevens, J. S., Towler, M. \& Wood, P. A. (2020). J. Appl. Cryst. 53, 226-235.

McNamara, L. E., Rill, T. A., Huckaba, A. J., Ganeshraj, V., Gayton, J., Nelson, R. A., Sharpe, E. A., Dass, A., Hammer, N. I. \& Delcamp, J. H. (2017). Chem. Eur. J. 23, 12494-12501.

Mederski, W., Beier, N., Burgdorf, L. T., Gericke, R., Klein, M. \& Tsaklakidis, C. (2012). US Patent 8(106,067 B2).

Mishra, B. B. \& Tiwari, V. K. (2011). Opportunity Challenge and Scope of Natural Products in Medicinal Chemistry, 1-61.

Qian, B.-H., Liu, W.-W., Lu, L.-D. \& Hu, H.-W. (2006). Acta Cryst. E62, o2363-o2364.

Sandeep, C., Padmashali, B., Venugopala, K. N., Kulkarni, R. S., Venugopala, R. \& Odhav, B. (2016a). Asian J. Chem. 28, 10431048.

Sandeep, C., Venugopala, K. N., Gleiser, R. M., Chetram, A., Padmashali, B., Kulkarni, R. S., Venugopala, R. \& Odhav, B. (2016b). Chem. Biol. Drug Des. 88, 899-904.

Sheldrick, G. M. (2008). Acta Cryst. A64, 112-122.

Sheldrick, G. M. (2015). Acta Cryst. C71, 3-8.

Sonnenschein, H. \& Schneider, M. (1997). Z. Kristallogr. New Cryst. Struct. 212, 161-162.

Spackman, M. A. \& Jayatilaka, D. (2009). CrystEngComm, 11, 19-32.

Spek, A. L. (2020). Acta Cryst. E76, 1-11.

Tafeenkov, V. A. \& Aslanov, L. A. (1980). Zh. Strukt. Khim. 21, 6978.

Tafeenkov, V. A. \& Au, O. (1996). Zh. Strukt. Khim. 37, 1181-1185.

Vaught, J. L., Carson, J. R., Carmosin, R. J., Blum, P. S., Persico, F. J., Hageman, W. E., Shank, R. P. \& Raffa, R. B. (1990). J. Pharmacol. Exp. Ther. 255, 1-10.

Venugopala, K. N., Tratrat, C., Pillay, M., Mahomoodally, F. M., Bhandary, S., Chopra, D., Morsy, M. A., Haroun, M., Aldhubiab, B. E., Attimarad, M., Nair, A. B., Sreeharsha, N., Venugopala, R., Chandrashekharappa, S., Alwassil, O. I. \& Odhav, B. (2019). Antibiotics, 8, 247-263.

Wolff, S. K., Grimwood, D. J., McKinnon, J. J., Turner, M. J., Jayatilaka, D. \& Spackman, M. A. (2012). CrystalExplorer12.5. University of Western Australia, Perth. 


\section{supporting information}

Acta Cryst. (2020). E76, 567-571 [https://doi.org/10.1107/S2056989020003837]

Structural investigation of methyl 3-(4-fluorobenzoyl)-7-methyl-2-phenyl-

indolizine-1-carboxylate, an inhibitory drug towards Mycobacterium

tuberculosis

\section{Avantika Hasija, Subhrajyoti Bhandary, Katharigatta N. Venugopala, Sandeep \\ Chandrashekharappa and Deepak Chopra}

Computing details

Data collection: APEX2 (Bruker, 2012); cell refinement: SAINT (Bruker, 2008); data reduction: SAINT (Bruker, 2008); program(s) used to solve structure: SHELXS97 (Sheldrick, 2008); program(s) used to refine structure: SHELXL2014 (Sheldrick, 2015); molecular graphics: X-SEED (Barbour, 2001) and Mercury (Macrae et al., 2020); software used to prepare material for publication: SHELXL2014 (Sheldrick, 2015) and PLATON (Spek, 2020).

3-(4-Fluorobenzoyl)-7-methyl-2-phenylindolizine-1-carboxylate

Crystal data

$\mathrm{C}_{24} \mathrm{H}_{18} \mathrm{FNO}_{3}$

$M_{r}=387.39$

Monoclinic, $P 2_{1} / n$

$a=7.3246(11) \AA$

$b=9.8460(13) \AA$

$c=25.837$ (4) $\AA$

$\beta=93.318(3)^{\circ}$

$V=1860.2(5) \AA^{3}$

$Z=4$

Data collection

Bruker Kappa Duo APEXII diffractometer

Radiation source: fine-focus sealed tube $0.5^{\circ} \varphi$ scans and $\omega$ scans Absorption correction: multi-scan

(SADABS; Bruker, 2008)

$T_{\min }=0.855, T_{\max }=1.000$

27523 measured reflections

\section{Refinement}

Refinement on $F^{2}$

Least-squares matrix: full

$R\left[F^{2}>2 \sigma\left(F^{2}\right)\right]=0.050$

$w R\left(F^{2}\right)=0.131$

$S=1.00$

4296 reflections

265 parameters
$F(000)=808$

$D_{\mathrm{x}}=1.383 \mathrm{Mg} \mathrm{m}^{-3}$

Mo $K \alpha$ radiation, $\lambda=0.71073 \AA$

Cell parameters from 27523 reflections

$\theta=2.2-27.6^{\circ}$

$\mu=0.10 \mathrm{~mm}^{-1}$

$T=173 \mathrm{~K}$

Block, yellow

$0.32 \times 0.18 \times 0.04 \mathrm{~mm}$

4296 independent reflections

2641 reflections with $I>2 \sigma(I)$

$R_{\text {int }}=0.090$

$\theta_{\max }=27.6^{\circ}, \theta_{\min }=2.2^{\circ}$

$h=-9 \rightarrow 9$

$k=-12 \rightarrow 12$

$l=-33 \rightarrow 33$

0 restraints

Primary atom site location: structure-invariant direct methods

Secondary atom site location: difference Fourier map

Hydrogen site location: inferred from neighbouring sites 
H-atom parameters constrained

$w=1 /\left[\sigma^{2}\left(F_{\mathrm{o}}^{2}\right)+(0.0555 P)^{2}+0.455 P\right]$

where $P=\left(F_{\mathrm{o}}^{2}+2 F_{\mathrm{c}}^{2}\right) / 3$

$(\Delta / \sigma)_{\max }<0.001$

$\Delta \rho_{\max }=0.29{\mathrm{e} \AA^{-3}}^{-3}$
$\Delta \rho_{\min }=-0.30$ e $\AA^{-3}$

Extinction correction: SHELXL-2014/7

(Sheldrick 2015,

$\mathrm{Fc}^{*}=\mathrm{kFc}\left[1+0.001 \times \mathrm{xF}^{2} \lambda^{3} / \sin (2 \theta)\right]^{-1 / 4}$

Extinction coefficient: 0.0044 (8)

Special details

Geometry. All esds (except the esd in the dihedral angle between two 1.s. planes) are estimated using the full covariance matrix. The cell esds are taken into account individually in the estimation of esds in distances, angles and torsion angles; correlations between esds in cell parameters are only used when they are defined by crystal symmetry. An approximate (isotropic) treatment of cell esds is used for estimating esds involving l.s. planes.

Fractional atomic coordinates and isotropic or equivalent isotropic displacement parameters $\left(\AA^{2}\right)$

\begin{tabular}{|c|c|c|c|c|}
\hline & $x$ & $y$ & $z$ & $U_{\text {iso }} * / U_{\text {eq }}$ \\
\hline F1 & $0.4480(2)$ & $0.70181(14)$ & $0.32107(5)$ & $0.0563(4)$ \\
\hline $\mathrm{O} 1$ & $0.2787(2)$ & $0.69998(15)$ & 0.00174 & $0.0381(4)$ \\
\hline $\mathrm{O} 2$ & 0.1668 (2) & $0.53237(14)$ & $-0.04937(5)$ & $0.0302(4)$ \\
\hline $\mathrm{O} 3$ & $0.5350(2)$ & $0.21447(15)$ & $0.16777(5)$ & $0.0336(4)$ \\
\hline N1 & $0.3494(2)$ & $0.26669(16)$ & $0.06884(6)$ & $0.0230(4)$ \\
\hline $\mathrm{C} 1$ & 0.3588 & $0.1276(2)$ & $0.07210(8)$ & $0.0293(5)$ \\
\hline H1 & 0.4025 & 0.0857 & 0.1035 & $0.035^{*}$ \\
\hline $\mathrm{C} 2$ & 0.3057 (3) & $0.0502(2)$ & $0.03060(8)$ & $0.0294(5)$ \\
\hline $\mathrm{H} 2$ & 0.3148 & -0.0459 & 0.0330 & $0.035^{*}$ \\
\hline $\mathrm{C} 3$ & 0.2368 & $0.1106(2)$ & $-0.01642(8)$ & $0.0269(5)$ \\
\hline $\mathrm{C} 4$ & $0.2282(3)$ & $0.2488(2)$ & $-0.01917(7)$ & $0.0252(5)$ \\
\hline H4 & 0.1830 & 0.2906 & -0.0505 & $0.030 *$ \\
\hline $\mathrm{C} 5$ & $0.2850(3)$ & $0.3307(2)$ & $0.02346(7)$ & $0.0232(4)$ \\
\hline C6 & $0.2913(3)$ & $0.4722(2)$ & $0.03281(7)$ & $0.0232(4)$ \\
\hline $\mathrm{C} 7$ & $0.3565(3)$ & $0.4924(2)$ & $0.08437(7)$ & $0.0225(4)$ \\
\hline $\mathrm{C} 8$ & $0.3910(3)$ & $0.3647(2)$ & $0.10738(7)$ & $0.0229(4)$ \\
\hline C9 & $0.1754(3)$ & $0.0225(2)$ & $-0.06142(8)$ & $0.0356(5)$ \\
\hline H9A & 0.1001 & 0.0760 & -0.0865 & $0.053^{*}$ \\
\hline H9B & 0.1035 & -0.0537 & -0.0491 & $0.053^{*}$ \\
\hline $\mathrm{H} 9 \mathrm{C}$ & 0.2826 & -0.0125 & -0.0781 & $0.053 *$ \\
\hline $\mathrm{C} 10$ & 0.2478 & $0.5806(2)$ & $-0.00470(7)$ & $0.0248(5)$ \\
\hline C11 & 0.1182 & $0.6340(2)$ & $-0.08794(8)$ & $0.0315(5)$ \\
\hline H11A & 0.0323 & 0.6988 & -0.0739 & $0.047^{*}$ \\
\hline H11B & 0.0606 & 0.5902 & -0.1188 & $0.047^{*}$ \\
\hline $\mathrm{H} 11 \mathrm{C}$ & 0.2285 & 0.6822 & -0.0974 & $0.047^{*}$ \\
\hline $\mathrm{C} 12$ & $0.3881(3)$ & $0.6250(2)$ & $0.11063(7)$ & $0.0250(5)$ \\
\hline $\mathrm{C} 13$ & $0.5610(3)$ & $0.6581(2)$ & $0.13180(7)$ & $0.0287(5)$ \\
\hline H13 & 0.6610 & 0.5989 & 0.1269 & $0.034 *$ \\
\hline $\mathrm{C} 14$ & 0.5889 & $0.7767(2)$ & $0.15993(8)$ & $0.0363(6)$ \\
\hline H14 & 0.7076 & 0.7984 & 0.1743 & $0.044 *$ \\
\hline C15 & 0.4445 & $0.8634(2)$ & $0.16707(8)$ & $0.0387(6)$ \\
\hline H15 & 0.4631 & 0.9443 & 0.1867 & $0.046^{*}$ \\
\hline $\mathrm{C} 16$ & 0.2724 & $0.8320(2)$ & $0.14543(8)$ & $0.0390(6)$ \\
\hline H16 & 0.1729 & 0.8918 & 0.1502 & $0.047 *$ \\
\hline
\end{tabular}




$\begin{array}{lllll}\mathrm{C} 17 & 0.2443(3) & 0.7144(2) & 0.11693(8) & 0.0326(5) \\ \mathrm{H} 17 & 0.1263 & 0.6945 & 0.1016 & 0.039^{*} \\ \mathrm{C} 18 & 0.4633(3) & 0.3265(2) & 0.15900(7) & 0.0253(5) \\ \mathrm{C} 19 & 0.4534(3) & 0.4247(2) & 0.20269(7) & 0.0257(5) \\ \mathrm{C} 20 & 0.6087(3) & 0.4441(2) & 0.23563(8) & 0.0324(5) \\ \mathrm{H} 20 & 0.7157 & 0.3924 & 0.2307 & 0.039^{*} \\ \mathrm{C} 21 & 0.6082(3) & 0.5381(2) & 0.27542(8) & 0.0385(6) \\ \mathrm{H} 21 & 0.7144 & 0.5534 & 0.2975 & 0.046^{*} \\ \mathrm{C} 22 & 0.4491(4) & 0.6086(2) & 0.28191(8) & 0.0374(6) \\ \mathrm{C} 23 & 0.2923(3) & 0.5899(2) & 0.25163(8) & 0.0328(5) \\ \mathrm{H} 23 & 0.1843 & 0.6393 & 0.2579 & 0.039^{*} \\ \mathrm{C} 24 & 0.2955(3) & 0.4967(2) & 0.21143(8) & 0.0283(5) \\ \mathrm{H} 24 & 0.1883 & 0.4820 & 0.1897 & 0.034^{*}\end{array}$

Atomic displacement parameters $\left(\AA^{2}\right)$

\begin{tabular}{|c|c|c|c|c|c|c|}
\hline & $U^{11}$ & $U^{22}$ & $U^{33}$ & $U^{12}$ & $U^{13}$ & $U^{23}$ \\
\hline $\mathrm{F} 1$ & $0.0812(12)$ & $0.0445(9)$ & $0.0415(8)$ & $0.0051(8)$ & $-0.0116(7)$ & $-0.0201(7)$ \\
\hline $\mathrm{O} 1$ & $0.0596(11)$ & $0.0203(8)$ & $0.0333(8)$ & $-0.0034(7)$ & $-0.0062(7)$ & $0.0057(7)$ \\
\hline $\mathrm{O} 2$ & $0.0413(9)$ & $0.0238(8)$ & $0.0248(7)$ & $0.0018(7)$ & $-0.0048(6)$ & $0.0033(6)$ \\
\hline $\mathrm{O} 3$ & $0.0420(9)$ & $0.0259(8)$ & $0.0323(8)$ & $0.0073(7)$ & $-0.0035(7)$ & $0.0032(7)$ \\
\hline N1 & $0.0271(10)$ & $0.0201(9)$ & $0.0219(8)$ & $0.0005(7)$ & $0.0025(7)$ & $0.0018(7)$ \\
\hline $\mathrm{C} 1$ & $0.0363(13)$ & $0.0213(11)$ & $0.0304(11)$ & $0.0029(9)$ & $0.0019(9)$ & $0.0052(9)$ \\
\hline $\mathrm{C} 2$ & $0.0369(13)$ & $0.0192(11)$ & $0.0324(11)$ & $0.0007(9)$ & $0.0036(9)$ & $-0.0004(9)$ \\
\hline $\mathrm{C} 3$ & $0.0282(11)$ & $0.0251(12)$ & $0.0280(11)$ & $-0.0045(9)$ & $0.0057(9)$ & $-0.0019(9)$ \\
\hline $\mathrm{C} 4$ & $0.0282(11)$ & $0.0248(11)$ & $0.0228(10)$ & $-0.0010(9)$ & $0.0020(8)$ & $0.0008(8)$ \\
\hline $\mathrm{C} 5$ & $0.0235(10)$ & $0.0234(11)$ & $0.0228(10)$ & $0.0005(8)$ & $0.0032(8)$ & $0.0033(8)$ \\
\hline C6 & $0.0255(11)$ & $0.0214(10)$ & $0.0228(10)$ & $0.0001(8)$ & $0.0020(8)$ & $0.0010(8)$ \\
\hline $\mathrm{C} 7$ & $0.0239(11)$ & $0.0206(10)$ & $0.0233(10)$ & $0.0014(8)$ & $0.0028(8)$ & $0.0005(8)$ \\
\hline $\mathrm{C} 8$ & $0.0260(11)$ & $0.0207(10)$ & $0.0219(9)$ & $0.0001(8)$ & $0.0021(8)$ & $-0.0014(8)$ \\
\hline C9 & $0.0460(14)$ & $0.0281(12)$ & $0.0326(12)$ & $-0.0038(10)$ & $0.0005(10)$ & $-0.0039(10)$ \\
\hline $\mathrm{C} 10$ & $0.0268(11)$ & $0.0243(11)$ & $0.0237(10)$ & $0.0000(9)$ & $0.0033(8)$ & $0.0015(9)$ \\
\hline C11 & $0.0382(13)$ & $0.0302(12)$ & $0.0256(10)$ & $0.0028(10)$ & $-0.0036(9)$ & $0.0077(9)$ \\
\hline $\mathrm{C} 12$ & $0.0373(12)$ & $0.0183(10)$ & $0.0197(9)$ & $-0.0001(9)$ & $0.0028(9)$ & $0.0029(8)$ \\
\hline $\mathrm{C} 13$ & $0.0375(12)$ & $0.0237(11)$ & $0.0253(10)$ & $-0.0035(9)$ & $0.0056(9)$ & $0.0008(9)$ \\
\hline $\mathrm{C} 14$ & $0.0494(15)$ & $0.0283(12)$ & $0.0311(12)$ & $-0.0106(11)$ & $0.0033(11)$ & $-0.0014(10)$ \\
\hline $\mathrm{C} 15$ & $0.0653(17)$ & $0.0199(12)$ & $0.0311(12)$ & $-0.0025(11)$ & $0.0026(11)$ & $-0.0030(10)$ \\
\hline $\mathrm{C} 16$ & $0.0589(16)$ & $0.0251(12)$ & $0.0333(12)$ & $0.0136(11)$ & $0.0062(11)$ & $0.0012(10)$ \\
\hline $\mathrm{C} 17$ & $0.0407(13)$ & $0.0267(12)$ & $0.0301(11)$ & $0.0059(10)$ & $-0.0009(10)$ & $0.0018(9)$ \\
\hline $\mathrm{C} 18$ & $0.0254(11)$ & $0.0259(11)$ & $0.0247(10)$ & $-0.0020(9)$ & $0.0026(8)$ & $0.0024(9)$ \\
\hline C19 & $0.0344(12)$ & $0.0223(11)$ & $0.0202(9)$ & $-0.0025(9)$ & $0.0000(9)$ & $0.0045(8)$ \\
\hline $\mathrm{C} 20$ & $0.0371(13)$ & $0.0289(12)$ & $0.0305(11)$ & $0.0013(10)$ & $-0.0046(10)$ & $0.0022(10)$ \\
\hline $\mathrm{C} 21$ & $0.0498(16)$ & $0.0324(13)$ & $0.0316(12)$ & $-0.0036(11)$ & $-0.0130(11)$ & $0.0016(10)$ \\
\hline $\mathrm{C} 22$ & $0.0614(17)$ & $0.0235(12)$ & $0.0268(11)$ & $-0.0004(11)$ & $-0.0024(11)$ & $-0.0027(9)$ \\
\hline $\mathrm{C} 23$ & $0.0420(14)$ & $0.0298(12)$ & $0.0269(11)$ & $0.0020(10)$ & $0.0058(10)$ & $0.0018(9)$ \\
\hline $\mathrm{C} 24$ & $0.0346(12)$ & $0.0274(12)$ & $0.0228(10)$ & $-0.0028(9)$ & $0.0010(9)$ & $0.0018(9)$ \\
\hline
\end{tabular}


Geometric parameters $\left(\AA,{ }^{\circ}\right)$

\begin{tabular}{|c|c|c|c|}
\hline $\mathrm{F} 1-\mathrm{C} 22$ & $1.367(2)$ & $\mathrm{C} 11-\mathrm{H} 11 \mathrm{~A}$ & 0.9800 \\
\hline $\mathrm{O} 1-\mathrm{C} 10$ & $1.206(2)$ & $\mathrm{C} 11-\mathrm{H} 11 \mathrm{~B}$ & 0.9800 \\
\hline $\mathrm{O} 2-\mathrm{C} 10$ & $1.353(2)$ & $\mathrm{C} 11-\mathrm{H} 11 \mathrm{C}$ & 0.9800 \\
\hline $\mathrm{O} 2-\mathrm{C} 11$ & $1.442(2)$ & $\mathrm{C} 12-\mathrm{C} 13$ & $1.389(3)$ \\
\hline $\mathrm{O} 3-\mathrm{C} 18$ & $1.236(2)$ & $\mathrm{C} 12-\mathrm{C} 17$ & $1.389(3)$ \\
\hline $\mathrm{N} 1-\mathrm{C} 1$ & $1.373(3)$ & $\mathrm{C} 13-\mathrm{C} 14$ & $1.384(3)$ \\
\hline $\mathrm{N} 1-\mathrm{C} 5$ & $1.389(2)$ & $\mathrm{C} 13-\mathrm{H} 13$ & 0.9500 \\
\hline $\mathrm{N} 1-\mathrm{C} 8$ & $1.408(2)$ & $\mathrm{C} 14-\mathrm{C} 15$ & $1.380(3)$ \\
\hline $\mathrm{C} 1-\mathrm{C} 2$ & $1.354(3)$ & $\mathrm{C} 14-\mathrm{H} 14$ & 0.9500 \\
\hline $\mathrm{C} 1-\mathrm{H} 1$ & 0.9500 & $\mathrm{C} 15-\mathrm{C} 16$ & $1.384(3)$ \\
\hline $\mathrm{C} 2-\mathrm{C} 3$ & $1.419(3)$ & C15-H15 & 0.9500 \\
\hline $\mathrm{C} 2-\mathrm{H} 2$ & 0.9500 & $\mathrm{C} 16-\mathrm{C} 17$ & $1.382(3)$ \\
\hline $\mathrm{C} 3-\mathrm{C} 4$ & $1.364(3)$ & $\mathrm{C} 16-\mathrm{H} 16$ & 0.9500 \\
\hline $\mathrm{C} 3-\mathrm{C} 9$ & $1.499(3)$ & C17-H17 & 0.9500 \\
\hline $\mathrm{C} 4-\mathrm{C} 5$ & $1.409(3)$ & $\mathrm{C} 18-\mathrm{C} 19$ & $1.491(3)$ \\
\hline $\mathrm{C} 4-\mathrm{H} 4$ & 0.9500 & $\mathrm{C} 19-\mathrm{C} 24$ & $1.387(3)$ \\
\hline $\mathrm{C} 5-\mathrm{C} 6$ & $1.414(3)$ & $\mathrm{C} 19-\mathrm{C} 20$ & $1.393(3)$ \\
\hline $\mathrm{C} 6-\mathrm{C} 7$ & $1.403(3)$ & $\mathrm{C} 20-\mathrm{C} 21$ & $1.383(3)$ \\
\hline $\mathrm{C} 6-\mathrm{C} 10$ & $1.465(3)$ & $\mathrm{C} 20-\mathrm{H} 20$ & 0.9500 \\
\hline $\mathrm{C} 7-\mathrm{C} 8$ & $1.407(3)$ & $\mathrm{C} 21-\mathrm{C} 22$ & $1.375(3)$ \\
\hline $\mathrm{C} 7-\mathrm{C} 12$ & $1.483(3)$ & $\mathrm{C} 21-\mathrm{H} 21$ & 0.9500 \\
\hline $\mathrm{C} 8-\mathrm{C} 18$ & $1.456(3)$ & $\mathrm{C} 22-\mathrm{C} 23$ & $1.364(3)$ \\
\hline C9-H9A & 0.9800 & $\mathrm{C} 23-\mathrm{C} 24$ & $1.388(3)$ \\
\hline C9- & 0.9800 & $\mathrm{C} 23-\mathrm{H} 23$ & 0.9500 \\
\hline $\mathrm{C} 9-\mathrm{H} 9 \mathrm{C}$ & 0.9800 & $\mathrm{C} 24-\mathrm{H} 24$ & 0.9500 \\
\hline $\mathrm{C} 10-\mathrm{O} 2-\mathrm{C} 11$ & $115.09(16)$ & $\mathrm{H} 11 \mathrm{~A}-\mathrm{C} 11-\mathrm{H} 11 \mathrm{C}$ & 109.5 \\
\hline $\mathrm{C} 1-\mathrm{N} 1-\mathrm{C} 5$ & $121.17(17)$ & $\mathrm{H} 11 \mathrm{~B}-\mathrm{C} 11-\mathrm{H} 11 \mathrm{C}$ & 109.5 \\
\hline $\mathrm{C} 1-\mathrm{N} 1-\mathrm{C} 8$ & $129.22(17)$ & $\mathrm{C} 13-\mathrm{C} 12-\mathrm{C} 17$ & $119.06(19)$ \\
\hline $\mathrm{C} 5-\mathrm{N} 1-\mathrm{C} 8$ & $109.56(16)$ & $\mathrm{C} 13-\mathrm{C} 12-\mathrm{C} 7$ & $120.09(18)$ \\
\hline $\mathrm{C} 2-\mathrm{C} 1-\mathrm{N} 1$ & $120.09(19)$ & $\mathrm{C} 17-\mathrm{C} 12-\mathrm{C} 7$ & $120.76(19)$ \\
\hline $\mathrm{C} 2-\mathrm{C} 1-\mathrm{H} 1$ & 120.0 & $\mathrm{C} 14-\mathrm{C} 13-\mathrm{C} 12$ & $120.5(2)$ \\
\hline $\mathrm{N} 1-\mathrm{C} 1-\mathrm{H} 1$ & 120.0 & $\mathrm{C} 14-\mathrm{C} 13-\mathrm{H} 13$ & 119.7 \\
\hline $\mathrm{C} 1-\mathrm{C} 2-\mathrm{C} 3$ & $120.92(19)$ & $\mathrm{C} 12-\mathrm{C} 13-\mathrm{H} 13$ & 119.7 \\
\hline $\mathrm{C} 1-\mathrm{C} 2-\mathrm{H} 2$ & 119.5 & $\mathrm{C} 15-\mathrm{C} 14-\mathrm{C} 13$ & $120.1(2)$ \\
\hline $\mathrm{C} 3-\mathrm{C} 2-\mathrm{H} 2$ & 119.5 & $\mathrm{C} 15-\mathrm{C} 14-\mathrm{H} 14$ & 120.0 \\
\hline $\mathrm{C} 4-\mathrm{C} 3-\mathrm{C} 2$ & $118.41(18)$ & $\mathrm{C} 13-\mathrm{C} 14-\mathrm{H} 14$ & 120.0 \\
\hline $\mathrm{C} 4-\mathrm{C} 3-\mathrm{C} 9$ & $121.74(19)$ & $\mathrm{C} 14-\mathrm{C} 15-\mathrm{C} 16$ & $119.7(2)$ \\
\hline $\mathrm{C} 2-\mathrm{C} 3-\mathrm{C} 9$ & $119.85(18)$ & $\mathrm{C} 14-\mathrm{C} 15-\mathrm{H} 15$ & 120.2 \\
\hline $\mathrm{C} 3-\mathrm{C} 4-\mathrm{C} 5$ & $121.33(19)$ & $\mathrm{C} 16-\mathrm{C} 15-\mathrm{H} 15$ & 120.2 \\
\hline $\mathrm{C} 3-\mathrm{C} 4-\mathrm{H} 4$ & 119.3 & $\mathrm{C} 17-\mathrm{C} 16-\mathrm{C} 15$ & $120.4(2)$ \\
\hline $\mathrm{C} 5-\mathrm{C} 4-\mathrm{H} 4$ & 119.3 & $\mathrm{C} 17-\mathrm{C} 16-\mathrm{H} 16$ & 119.8 \\
\hline $\mathrm{N} 1-\mathrm{C} 5-\mathrm{C} 4$ & $118.07(18)$ & $\mathrm{C} 15-\mathrm{C} 16-\mathrm{H} 16$ & 119.8 \\
\hline $\mathrm{N} 1-\mathrm{C} 5-\mathrm{C} 6$ & $107.26(16)$ & $\mathrm{C} 16-\mathrm{C} 17-\mathrm{C} 12$ & $120.2(2)$ \\
\hline $\mathrm{C} 4-\mathrm{C} 5-\mathrm{C} 6$ & $134.66(18)$ & $\mathrm{C} 16-\mathrm{C} 17-\mathrm{H} 17$ & 119.9 \\
\hline $\mathrm{C} 7-\mathrm{C} 6-\mathrm{C} 5$ & $107.94(17)$ & $\mathrm{C} 12-\mathrm{C} 17-\mathrm{H} 17$ & 119.9 \\
\hline
\end{tabular}




\begin{tabular}{|c|c|c|c|}
\hline $\mathrm{C} 7-\mathrm{C} 6-\mathrm{C} 10$ & $125.01(18)$ & $\mathrm{O} 3-\mathrm{C} 18-\mathrm{C} 8$ & $121.78(18)$ \\
\hline $\mathrm{C} 5-\mathrm{C} 6-\mathrm{C} 10$ & $126.97(17)$ & $\mathrm{O} 3-\mathrm{C} 18-\mathrm{C} 19$ & $118.57(17)$ \\
\hline $\mathrm{C} 6-\mathrm{C} 7-\mathrm{C} 8$ & $108.49(17)$ & $\mathrm{C} 8-\mathrm{C} 18-\mathrm{C} 19$ & 119.64 (17) \\
\hline $\mathrm{C} 6-\mathrm{C} 7-\mathrm{C} 12$ & $126.51(17)$ & $\mathrm{C} 24-\mathrm{C} 19-\mathrm{C} 20$ & $119.31(19)$ \\
\hline $\mathrm{C} 8-\mathrm{C} 7-\mathrm{C} 12$ & $124.99(17)$ & $\mathrm{C} 24-\mathrm{C} 19-\mathrm{C} 18$ & $122.16(18)$ \\
\hline $\mathrm{C} 7-\mathrm{C} 8-\mathrm{N} 1$ & $106.72(16)$ & $\mathrm{C} 20-\mathrm{C} 19-\mathrm{C} 18$ & 118.54 (19) \\
\hline $\mathrm{C} 7-\mathrm{C} 8-\mathrm{C} 18$ & $131.69(18)$ & $\mathrm{C} 21-\mathrm{C} 20-\mathrm{C} 19$ & $120.6(2)$ \\
\hline $\mathrm{N} 1-\mathrm{C} 8-\mathrm{C} 18$ & $121.52(17)$ & $\mathrm{C} 21-\mathrm{C} 20-\mathrm{H} 20$ & 119.7 \\
\hline $\mathrm{C} 3-\mathrm{C} 9-\mathrm{H} 9 \mathrm{~A}$ & 109.5 & $\mathrm{C} 19-\mathrm{C} 20-\mathrm{H} 20$ & 119.7 \\
\hline C3- $\mathrm{C} 9-\mathrm{H} 9 \mathrm{~B}$ & 109.5 & $\mathrm{C} 22-\mathrm{C} 21-\mathrm{C} 20$ & $117.8(2)$ \\
\hline $\mathrm{H} 9 \mathrm{~A}-\mathrm{C} 9-\mathrm{H} 9 \mathrm{~B}$ & 109.5 & $\mathrm{C} 22-\mathrm{C} 21-\mathrm{H} 21$ & 121.1 \\
\hline $\mathrm{C} 3-\mathrm{C} 9-\mathrm{H} 9 \mathrm{C}$ & 109.5 & $\mathrm{C} 20-\mathrm{C} 21-\mathrm{H} 21$ & 121.1 \\
\hline $\mathrm{H} 9 \mathrm{~A}-\mathrm{C} 9-\mathrm{H} 9 \mathrm{C}$ & 109.5 & $\mathrm{C} 23-\mathrm{C} 22-\mathrm{F} 1$ & $118.3(2)$ \\
\hline $\mathrm{H} 9 \mathrm{~B}-\mathrm{C} 9-\mathrm{H} 9 \mathrm{C}$ & 109.5 & $\mathrm{C} 23-\mathrm{C} 22-\mathrm{C} 21$ & $123.6(2)$ \\
\hline $\mathrm{O} 1-\mathrm{C} 10-\mathrm{O} 2$ & $121.96(18)$ & $\mathrm{F} 1-\mathrm{C} 22-\mathrm{C} 21$ & $118.1(2)$ \\
\hline $\mathrm{O} 1-\mathrm{C} 10-\mathrm{C} 6$ & $125.95(18)$ & $\mathrm{C} 22-\mathrm{C} 23-\mathrm{C} 24$ & $117.9(2)$ \\
\hline $\mathrm{O} 2-\mathrm{C} 10-\mathrm{C} 6$ & $112.09(17)$ & $\mathrm{C} 22-\mathrm{C} 23-\mathrm{H} 23$ & 121.0 \\
\hline $\mathrm{O} 2-\mathrm{C} 11-\mathrm{H} 11 \mathrm{~A}$ & 109.5 & $\mathrm{C} 24-\mathrm{C} 23-\mathrm{H} 23$ & 121.0 \\
\hline $\mathrm{O} 2-\mathrm{C} 11-\mathrm{H} 11 \mathrm{~B}$ & 109.5 & $\mathrm{C} 19-\mathrm{C} 24-\mathrm{C} 23$ & $120.7(2)$ \\
\hline $\mathrm{H} 11 \mathrm{~A}-\mathrm{C} 11-\mathrm{H} 11 \mathrm{~B}$ & 109.5 & $\mathrm{C} 19-\mathrm{C} 24-\mathrm{H} 24$ & 119.6 \\
\hline $\mathrm{O} 2-\mathrm{C} 11-\mathrm{H} 11 \mathrm{C}$ & 109.5 & $\mathrm{C} 23-\mathrm{C} 24-\mathrm{H} 24$ & 119.6 \\
\hline $\mathrm{C} 5-\mathrm{N} 1-\mathrm{C} 1-\mathrm{C} 2$ & $0.4(3)$ & $\mathrm{C} 7-\mathrm{C} 6-\mathrm{C} 10-\mathrm{O} 2$ & $-172.44(18)$ \\
\hline $\mathrm{C} 8-\mathrm{N} 1-\mathrm{C} 1-\mathrm{C} 2$ & 177.53 (19) & $\mathrm{C} 5-\mathrm{C} 6-\mathrm{C} 10-\mathrm{O} 2$ & $11.1(3)$ \\
\hline $\mathrm{N} 1-\mathrm{C} 1-\mathrm{C} 2-\mathrm{C} 3$ & $-1.2(3)$ & $\mathrm{C} 6-\mathrm{C} 7-\mathrm{C} 12-\mathrm{C} 13$ & $-121.3(2)$ \\
\hline $\mathrm{C} 1-\mathrm{C} 2-\mathrm{C} 3-\mathrm{C} 4$ & $1.2(3)$ & $\mathrm{C} 8-\mathrm{C} 7-\mathrm{C} 12-\mathrm{C} 13$ & $57.4(3)$ \\
\hline $\mathrm{C} 1-\mathrm{C} 2-\mathrm{C} 3-\mathrm{C} 9$ & $-178.8(2)$ & $\mathrm{C} 6-\mathrm{C} 7-\mathrm{C} 12-\mathrm{C} 17$ & $62.2(3)$ \\
\hline $\mathrm{C} 2-\mathrm{C} 3-\mathrm{C} 4-\mathrm{C} 5$ & $-0.4(3)$ & $\mathrm{C} 8-\mathrm{C} 7-\mathrm{C} 12-\mathrm{C} 17$ & $-119.0(2)$ \\
\hline $\mathrm{C} 9-\mathrm{C} 3-\mathrm{C} 4-\mathrm{C} 5$ & $179.56(19)$ & $\mathrm{C} 17-\mathrm{C} 12-\mathrm{C} 13-\mathrm{C} 14$ & $1.7(3)$ \\
\hline $\mathrm{C} 1-\mathrm{N} 1-\mathrm{C} 5-\mathrm{C} 4$ & $0.4(3)$ & $\mathrm{C} 7-\mathrm{C} 12-\mathrm{C} 13-\mathrm{C} 14$ & $-174.86(18)$ \\
\hline $\mathrm{C} 8-\mathrm{N} 1-\mathrm{C} 5-\mathrm{C} 4$ & $-177.26(17)$ & $\mathrm{C} 12-\mathrm{C} 13-\mathrm{C} 14-\mathrm{C} 15$ & $-0.2(3)$ \\
\hline $\mathrm{C} 1-\mathrm{N} 1-\mathrm{C} 5-\mathrm{C} 6$ & $179.55(18)$ & $\mathrm{C} 13-\mathrm{C} 14-\mathrm{C} 15-\mathrm{C} 16$ & $-0.8(3)$ \\
\hline $\mathrm{C} 8-\mathrm{N} 1-\mathrm{C} 5-\mathrm{C} 6$ & $1.9(2)$ & $\mathrm{C} 14-\mathrm{C} 15-\mathrm{C} 16-\mathrm{C} 17$ & $0.2(3)$ \\
\hline $\mathrm{C} 3-\mathrm{C} 4-\mathrm{C} 5-\mathrm{N} 1$ & $-0.4(3)$ & $\mathrm{C} 15-\mathrm{C} 16-\mathrm{C} 17-\mathrm{C} 12$ & $1.3(3)$ \\
\hline $\mathrm{C} 3-\mathrm{C} 4-\mathrm{C} 5-\mathrm{C} 6$ & $-179.2(2)$ & $\mathrm{C} 13-\mathrm{C} 12-\mathrm{C} 17-\mathrm{C} 16$ & $-2.2(3)$ \\
\hline $\mathrm{N} 1-\mathrm{C} 5-\mathrm{C} 6-\mathrm{C} 7$ & $-1.1(2)$ & $\mathrm{C} 7-\mathrm{C} 12-\mathrm{C} 17-\mathrm{C} 16$ & $174.28(19)$ \\
\hline $\mathrm{C} 4-\mathrm{C} 5-\mathrm{C} 6-\mathrm{C} 7$ & $177.8(2)$ & $\mathrm{C} 7-\mathrm{C} 8-\mathrm{C} 18-\mathrm{O} 3$ & $-157.8(2)$ \\
\hline $\mathrm{N} 1-\mathrm{C} 5-\mathrm{C} 6-\mathrm{C} 10$ & $175.82(18)$ & $\mathrm{N} 1-\mathrm{C} 8-\mathrm{C} 18-\mathrm{O} 3$ & $19.0(3)$ \\
\hline $\mathrm{C} 4-\mathrm{C} 5-\mathrm{C} 6-\mathrm{C} 10$ & $-5.3(4)$ & $\mathrm{C} 7-\mathrm{C} 8-\mathrm{C} 18-\mathrm{C} 19$ & $21.5(3)$ \\
\hline $\mathrm{C} 5-\mathrm{C} 6-\mathrm{C} 7-\mathrm{C} 8$ & $0.0(2)$ & $\mathrm{N} 1-\mathrm{C} 8-\mathrm{C} 18-\mathrm{C} 19$ & $-161.76(18)$ \\
\hline $\mathrm{C} 10-\mathrm{C} 6-\mathrm{C} 7-\mathrm{C} 8$ & $-177.05(19)$ & $\mathrm{O} 3-\mathrm{C} 18-\mathrm{C} 19-\mathrm{C} 24$ & $-134.5(2)$ \\
\hline $\mathrm{C} 5-\mathrm{C} 6-\mathrm{C} 7-\mathrm{C} 12$ & $178.88(19)$ & $\mathrm{C} 8-\mathrm{C} 18-\mathrm{C} 19-\mathrm{C} 24$ & $46.2(3)$ \\
\hline $\mathrm{C} 10-\mathrm{C} 6-\mathrm{C} 7-\mathrm{C} 12$ & $1.9(3)$ & $\mathrm{O} 3-\mathrm{C} 18-\mathrm{C} 19-\mathrm{C} 20$ & $45.5(3)$ \\
\hline $\mathrm{C} 6-\mathrm{C} 7-\mathrm{C} 8-\mathrm{N} 1$ & $1.2(2)$ & $\mathrm{C} 8-\mathrm{C} 18-\mathrm{C} 19-\mathrm{C} 20$ & $-133.8(2)$ \\
\hline $\mathrm{C} 12-\mathrm{C} 7-\mathrm{C} 8-\mathrm{N} 1$ & $-177.78(18)$ & $\mathrm{C} 24-\mathrm{C} 19-\mathrm{C} 20-\mathrm{C} 21$ & $-2.6(3)$ \\
\hline $\mathrm{C} 6-\mathrm{C} 7-\mathrm{C} 8-\mathrm{C} 18$ & $178.3(2)$ & $\mathrm{C} 18-\mathrm{C} 19-\mathrm{C} 20-\mathrm{C} 21$ & 177.43 (19) \\
\hline $\mathrm{C} 12-\mathrm{C} 7-\mathrm{C} 8-\mathrm{C} 18$ & $-0.7(3)$ & $\mathrm{C} 19-\mathrm{C} 20-\mathrm{C} 21-\mathrm{C} 22$ & $1.4(3)$ \\
\hline $\mathrm{C} 1-\mathrm{N} 1-\mathrm{C} 8-\mathrm{C} 7$ & $-179.3(2)$ & $\mathrm{C} 20-\mathrm{C} 21-\mathrm{C} 22-\mathrm{C} 23$ & $0.6(3)$ \\
\hline
\end{tabular}




$\begin{array}{llll}\mathrm{C} 5-\mathrm{N} 1-\mathrm{C} 8-\mathrm{C} 7 & -1.9(2) & \mathrm{C} 20-\mathrm{C} 21-\mathrm{C} 22-\mathrm{F} 1 & -179.8(2) \\ \mathrm{C} 1-\mathrm{N} 1-\mathrm{C} 8-\mathrm{C} 18 & 3.2(3) & \mathrm{F} 1-\mathrm{C} 22-\mathrm{C} 23-\mathrm{C} 24 & 178.97(19) \\ \mathrm{C} 5-\mathrm{N} 1-\mathrm{C} 8-\mathrm{C} 18 & -179.35(17) & \mathrm{C} 21-\mathrm{C} 22-\mathrm{C} 23-\mathrm{C} 24 & -1.4(3) \\ \mathrm{C} 11-\mathrm{O} 2-\mathrm{C} 10-\mathrm{O} 1 & -1.0(3) & \mathrm{C} 20-\mathrm{C} 19-\mathrm{C} 24-\mathrm{C} 23 & 1.7(3) \\ \mathrm{C} 11-\mathrm{O} 2-\mathrm{C} 10-\mathrm{C} 6 & 179.33(17) & \mathrm{C} 18-\mathrm{C} 19-\mathrm{C} 24-\mathrm{C} 23 & -178.30(19) \\ \mathrm{C} 7-\mathrm{C} 6-\mathrm{C} 10-\mathrm{O} 1 & 7.9(3) & \mathrm{C} 22-\mathrm{C} 23-\mathrm{C} 24-\mathrm{C} 19 & 0.2(3) \\ \mathrm{C} 5-\mathrm{C} 6-\mathrm{C} 10-\mathrm{O} 1 & -168.6(2) & & \end{array}$

Hydrogen-bond geometry $\left(\AA,{ }^{\circ}\right)$

\begin{tabular}{lllll}
\hline$D-\mathrm{H} \cdots A$ & $D-\mathrm{H}$ & $\mathrm{H} \cdots A$ & $D \cdots A$ & $D-\mathrm{H} \cdots A$ \\
\hline $\mathrm{C} 1-\mathrm{H} 1 \cdots \mathrm{O} 3$ & 0.95 & 2.26 & $2.853(3)$ & 120 \\
$\mathrm{C} 4-\mathrm{H} 4 \cdots \mathrm{O} 2$ & 0.95 & 2.38 & $2.927(2)$ & 116 \\
$\mathrm{C} 21-\mathrm{H} 21 \cdots \mathrm{O}^{\mathrm{i}}$ & 0.95 & 2.54 & $3.399(3)$ & 149 \\
$\mathrm{C} 2-\mathrm{H} 2 \cdots{ }^{\mathrm{ii}}$ & 0.95 & 2.63 & $3.531(4)$ & 157 \\
$\mathrm{C} 15-\mathrm{H} 15 \cdots \mathrm{O} 3^{\mathrm{iii}}$ & 0.95 & 2.76 & $3.519(4)$ & 137 \\
$\mathrm{C} 1-\mathrm{H} 1 \cdots \mathrm{C} 15^{i i}$ & 0.95 & 2.74 & $3.6064(3)$ & 152 \\
$\mathrm{C} 11-\mathrm{H} 11 A^{\cdots} \cdots \mathrm{C}^{\text {iv }}$ & 0.98 & 2.74 & $3.4906(1)$ & 133 \\
$\mathrm{C} 11-\mathrm{H} 11 B \cdots \mathrm{F} 1^{\mathrm{v}}$ & 0.98 & 2.67 & $3.0585(3)$ & 104 \\
$\mathrm{C} 23-\mathrm{H} 23 \cdots 3^{\text {vi }}$ & 0.95 & 2.67 & $3.4875(3)$ & 143
\end{tabular}

Symmetry codes: (i) $-x+3 / 2, y+1 / 2,-z+1 / 2$; (ii) $x, y-1, z$; (iii) $x, y+1, z$; (iv) $-x,-y+1,-z$; (v) $x-1 / 2,-y+3 / 2, z-1 / 2$; (vi) $-x+1 / 2, y+1 / 2,-z+1 / 2$. 